traces, but these are small matters.

It is right and proper that the Foreword should contain a fulsome acknowledgement of the work of Merton and Morton, and almost every paper refers to the discoveries of this remarkable pair. Without them, techniques for non-invasive stimulation of the brain and cord would not exist.

CLARE J FOWLER

The Lennox-Gastaut Syndrome. Edited by Ernst Niedermeyer, Rolf Degen. (Pp 500; \$88.00.) New York: Alan R Liss, 1988.

This is the 45th volume of the Neurology and Neurobiology series, and the first in this series to be devoted entirely to epilepsy. It is in fact the proceedings of a conference held in Bad Kreuznach, in September 1987, concerned with many aspects of the Lennox Gastaut syndrome. The book consists of 30 chapters, by over 70 distinguished contributors from the world of paediatric neurology and epileptology. The Lennox Gastaut syndrome was first defined in 1966 and since then has been extensively discussed, although whether the constellation of signs constitutes a specific syndrome is questionable (an issue not addressed in this text). What does this book contribute? It undoubtably provides the most accessable source of reference material about the syndrome, and covers a wide range of topics. Some are important, recent, and not available in the English language literature, and are well covered here. As with so many published proceedings, however, there is much which is redundant or repetitive. The quality is variable, with some excellent chapters, for example on neuropathological and electrophysiological aspects, and some which are both weak and uncritical. The book is also notably poorly produced (even for a camera ready text), with mixtures of type faces, styles and formats; this does no justice to the editors or the authors, and is an abrogation of a publishers' art. This is for the shelves of an epilepsy specialist only, and one prepared to overlook the poor production quality.

SIMON SHORVON

\section{Diagnostic Manual of Tumours of the Central} Nervous System. By Anthony J Franks. (Pp 142; £45.00.) Edinburgh: Churchill Livingstone, 1988.

Of the florid production of neurological books we see nowadays, a large part is taken by those dealing with tumours of the nervous system. Dr Franks' effort Diagnostic Manual of Tumours of the Central Nervous System comes now into such a rich field and obviously will not avoid being compared with other, often highly valuable, publication on the same subject.

The book has been planned rather traditionally with an introductory chapter on techniques of preparation and staining of the tissues. Artefacts are common events when fresh preparations are used for diagnostic purposes and the author describes and illustrates them comparing the advantages and pitfalls of smears and frozen sections.

The tumours are subsequently presented according to the classical sequence; gliomas first, followed by the various meningeal tumours, primitive neuroectodermal and neuronal neoplasms, nerve sheath, reticular, vascular, pituitary, pineal, germ cell tumours. The last chapters describe epithelial tumours and maldevelopmental lesions, paraganglioma, chordoma and metastases. Each chapter consists of a standard description of the main morphological features of the various neoplasms accompanied by numerous good quality illustrations. In some cases, the reader has the chance of comparing the appearances of the same lesion in smear, frozen and paraffin preparations.

Having briefly described the layout of this book, it is less easy to comment on what useful and original information it has to offer to the readers that does not already exist in other publications. Since the manual was designed, in the words of the author, "to provide a practical bench manual for use by trainees ... and experienced pathologists . . .", I could find very little that could help in everyday diagnosis, in addition to standard descriptions and pictures. Nor does the recurring statement that "further sampling" be done help very much; while every pathologist is aware of the fact that more sections should be examined in order to make the best possible diagnosis, the present neurosurgical trend is towards performing more and more CT directed needle biopsies which give the pathologist very little tissue. Unfortunately, I could not see any comment on this subject.

Furthermore, I would have liked some more display of immunohistochemical expertise by a pathologist who has done a lot in this field; GFAP is frequently mentioned but other important markers are not sufficiently dealt with in the differential diagnosis; yet most laboratories have access to antibodies and some directive on the subject could have certainly helped to avoid misuse and misinterpretations.

In conclusion, a rather conventional book which can offer limited help to everyday neuropathological work. This, the few inaccuracies and the relatively high price are the main liabilities which are only marginally offset by a succinct test and nice pictures.

F SCARAVILLI

Evoked Potentials III: The Third International Evoked Potentials Symposium. Edited by C Barber, TBlum. (Pp 487; £108.00.) Guildford: Butterworth Scientific, 1988.

This well-produced volume contains selected papers from the Evoked Potentials Symposium which was held in West Berlin in late 1986. The most important and useful part of the book is the first section, termed Background and Perspective. This contains eight review articles by invited participants, and al are well worth attention from anyone with more than a passing interest in clinical neurophysiology. A clear summary of nearand far-field potentials and the junctional potential by Kimura is followed by añ idiosyncratic but enjoyable overview of auditory evoked potentials by Jewett, and@ then by Bodis-Wollner's paper on the up and coming N70 component of the VEP. Mauguièrés chapter on upper limb SEPs 응 and the possible role of mapping is characteristically clear and thorough, and as usuale induces feelings of guilt and resolutionsabout trying harder with non-cephalic references. Would that one had the time and the technicians for what is now routine in Lyons, but two runs of two thousand averages per side makes it a lengthy business. Event related potentials are reviewed, in as much as such a confusing mass of data can be briefly reviewed, by McCallum, and Allison summarises current views of aging and maturation of EPs, both short- and longlatency. Lehmann assesses EP mapping, with some comments on analysis thereof, and finally Kaufman and Williamson review some recent developments in the emerging field of magnetoencephalography, as applied to evoked potentials.

The rest of the volume comprises 60 papers chosen from those submitted and presented at the Symposium. There is the traditional section for each of: AEPs, VEPs, SEP, Cognitive Function and Multimodal studies, as well as five papers on the relatively new field of motor evoked potentials. Many of these papers, perhaps the VEP section in 
particular, will be of interest to most of those who read this book, though some, I fear, will not be of much interest to more than a few. Quite a lot have now appeared in the journals, though the authors are to be congratulated on the speed with which they have produced this book.

I think that there is enough in this book between the review articles, of value to all, and the rest, according to individual preference, for it to earn a place in the department library. Individuals who baulk at the cost should seriously consider obtaining it direct from the USA: at around half of the price cited here it is a much more attractive proposition.

NMF MURRAY

Elements of Petit Mal Epilepsy. Edited by M S Myslobodsky, A F Mirsky. (Pp 419; \$81.95.) New York: Peter Lang Publishing, 1989.

There can be no doubt that petit mal (simple absence) epilepsy represents one of the most fascinating of the epileptic syndromes. It is the most clearly identified of the genetically determined epilepsy syndromes and the one that is most easily identified by electroencephalographic (EEG) recordings. In spite of this, the genetics and neurophysiology of this condition remain controversial and so it was with a sense of some expectation that $I$ picked up this book which attempts to provide an up-to-date summary of research and theory of petit mal epilepsy.

The book is a multi-author production, the major contributions coming from a number of basic scientists and clinicians working in the National Institute of Mental Health/National Institute of Health, Bethesda, Maryland. Additional contributions are provided by Pierre Gloor, Elving Anderson and Allen Hauser. Some of the chapters are excellent, including those of the genetics of petit mal epilepsy, the mechanisms of generalised spike wave discharge and the anticonvulsant pharmacology of cultured CNS neurons. However, the remaining chapters are likely to be a disappointment for practising neurologists seeking further enlightenment in this area. Significant portions of the book are given over to long discussions of the effects of absence seizures on awareness and evoked potentials. As the arguments in these chapters develop it seems that the central relationship between generalised spike wave discharge and its clinical effects become evermore remote and the final conclusions are difficult to understand. The ultimate manifestation of this approach is a chapter which attempts to draw parallels between petit mal epilepsy and a number of metabolic encephalopathies including dialysis dementia and hepatic encephalopathy. Such an approach seems a final step into the realms of fantasy ignoring as it does the enormous clinical differences that manifestly exist between these conditions.

In the final analysis I cannot feel that the book has satisfactorily achieved its aims. Those chapters of a satisfactory and high standard are available in similar forms in other publications and the rest of the bulk of this volume will not be of major interest or have significant educational value to the majority of clinical neurologists. For this reason it is difficult to recommend this publication.

DW CHADWICK

Psychopharmacology and Reaction Time. Edited by I Hindmarch, B Aufdembrinke, $\mathbf{H}$ Ott. (Pp 194; £24.50). Chichester: John Wiley, 1988.

This book is based on a workshop on the use of reaction time in psychopharmacology that took place in West Germany in September 1985. It contains 14 chapters and includes contributions from a number of eminent workers in the field of information processing. In general, the majority of chapters focus on the approach that the author has found or considers most fruitful in the investigation of drug effects on reaction time, together with illustrative experimental evidence. Most chapters deal with the effects of drugs on the quality and speed of information processing, although a number consider the influence of other independent variables such as age, IQ, anxiety, and time of day on reaction time.

A number of salient conceptual issues and experimental approaches such as the distinction between structural processes and energetic mechanisms in human performance research, state-versus performance-oriented analysis, the individual as an active processor of information and the issue of speedaccuracy trade-off are discussed. Multidimensional assessment through recording of evoked potentials during reaction time tasks, the importance of qualitative examination of the pattern of responses in serial reaction time studies in addition to quantitative analysis, the distinction between liftoff time and movement time, the advantages of using a task that allows manipulation of task variables instead of a test battery, the superiority of ecologically valid, real-lifø tasks over laboratory tasks are some of the methodological refinements of reaction time measures that are highlighted in the book.

Unlike many books that are based on the proceedings of a workshop or conferences where the finished product often looks like $\Phi$ series of bound photocopies, this book professionally edited and produced. The only drawbacks are the complex style of writing in a number of chapters and the failure to describe specialised techniques in simple terms for the benefit of non-expeit readers. A noticeable example of the latter Sternberg's additive factor method, which referred to or experimentally-employed i 8 the majority of chapters and yet is nowhere described in any detail. Besides psychopharmacologists, the book is suitable for a wide readership. It will be of value to anyone engaged in or embarking in informatiog processing/reaction time research. At $£ 24.50$ this relatively slim volume is likely to be sought on the shelves of institution libraries rather than personally acquired. N MARJAN JAHANSHAH

\section{Correction}

In the paper by Cole, Philip, Sedgugiel "Stability and tremor in the fingers $\bar{D}$ associated with cerebellar hemisphere cerebellar tract lesions in man", ( $J$ Neuto Neurosurg Psychiatry 1988;51:558-68) figure lc was an unrelated MRI scan. The correc5 CT scan is shown below.

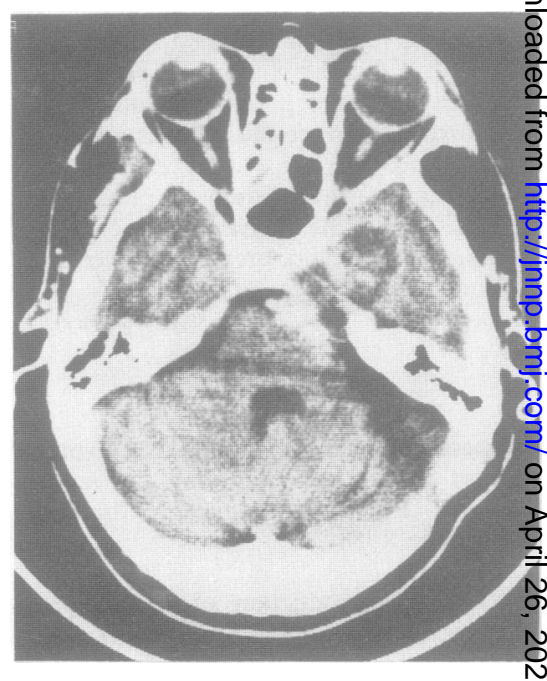

